\title{
BMJ Open A theory-based, task-oriented, outdoor walking programme for older adults with difficulty walking outdoors: protocol for the Getting Older Adults Outdoors (GO-OUT) randomised controlled trial
}

\author{
Nancy M Salbach, ${ }^{\oplus 1,2}$ Ruth Barclay, ${ }^{3}$ Sandra C Webber, ${ }^{3}$ C A Jones, ${ }^{4}$ \\ Nancy E Mayo, ${ }^{5}$ Lisa M Lix, ${ }^{6}$ Jacquie Ripat, ${ }^{7}$ Theresa Grant, ${ }^{8}$ Cornelia van Ineveld, ${ }^{9}$ \\ Philip D Chilibeck ${ }^{10}$
}

To cite: Salbach NM, Barclay R, Webber SC, et al. A theorybased, task-oriented, outdoor walking programme for older adults with difficulty walking outdoors: protocol for the Getting Older Adults Outdoors (GO-OUT) randomised controlled trial. BMJ Open 2019;9:e029393. doi:10.1136/ bmjopen-2019-029393

- Prepublication history for this paper is available online. To view these files please visit the journal online (http://dx.doi org/10.1136/bmjopen-2019029393).

Received 24 January 2019 Revised 17 February 2019 Accepted 20 February 2019
Check for updates

(C) Author(s) (or their employer(s)) 2019. Re-use permitted under CC BY-NC. No commercial re-use. See rights and permissions. Published by BMJ.

For numbered affiliations see end of article.

Correspondence to Dr Nancy M Salbach; nancy.salbach@utoronto.ca

\section{ABSTRACT}

Introduction A theory-based, task-oriented, community walking programme can increase outdoor walking activity among older adults to optimise functional independence, social participation and well-being. The study objective is to determine if there is a difference in the change in outdoor walking activity from baseline to 10 weeks, 5.5 months and 12 months after receiving a 1-day interactive workshop and outdoor walking programme (Getting Older Adults Outdoors (G0-OUT)) compared with the workshop and weekly reminders (WR) in older adults with difficulty walking outdoors.

Methods and analysis A randomised controlled trial is being conducted in four urban Canadian communities. We will stratify 240 individuals by site and participant type (ie, individual vs spousal/friend pair) and randomise to either the G0-OUT or WR intervention. The G0-OUT intervention involves a 1-day workshop, where participants complete eight interactive stations to build knowledge and skills to walk outside, followed by a 10-week group outdoor walking programme (two 1-hour sessions/week) led by a physiotherapist or kinesiologist in parks. The WR intervention consists of the same workshop and 10 weekly telephone reminders to facilitate outdoor walking. The primary outcome measure is mean outdoor walking time in minutes/week derived from accelerometry and global positioning system data. G0-OUT is powered to detect an effect size of 0.4 , given $\alpha=0.05, \beta=0.20$, equal number of participants/group and a $20 \%$ attrition rate. Secondary outcomes include physical activity, lifespace mobility, participation, health-related quality of life, balance, leg strength, walking self-efficacy, walking speed, walking distance/endurance and mood.

Ethics and dissemination G0-OUT has received ethics approval at all sites. A Data Safety Monitoring Board will monitor adverse events. We will disseminate findings through lay summaries, conference presentations and journal articles.

Trial registration number NCT03292510 (Pre-results).
Strengths and limitations of this study

- Conducting the study in four urban sites is expected to optimise external validity to urban centres variable in population size, weather conditions and environmental barriers but limit generalisability to rural centres.

- Use of accelerometry and global positioning system technology as well as self-reported methods represents a novel approach to estimating outdoor walking activity in older adults.

- Allowing individuals to participate on their own or with a spouse/friend optimises the ecological validity of the intervention.

- Participants cannot be blinded to the intervention they receive and this may introduce bias.

- A mixed methods process evaluation will increase understanding of intended and unintended consequences of the interventions, potent intervention components, mechanisms of effect and effect modification.

\section{INTRODUCTION}

An estimated $42 \%$ of older adults describe limited participation in outdoor walking defined as walking outside fewer than 3 days a week. ${ }^{1}$ Infrequent performance of outdoor walking is a marker of frailty ${ }^{2}$ and can increase the risk of mobility and self-care decline, social isolation and reduced health-related quality of life (HRQL). ${ }^{3}{ }^{4}$ Difficulty walking represents a key individual barrier to community mobility in older adults. ${ }^{5}$ Decreased fitness, balance and leg strength are physical factors that contribute to reductions in outdoor walking. ${ }^{26}$ Psychological factors, such as fear of moving outdoors and decreased 
self-efficacy in community mobility, may also limit engagement in outdoor walking among older adults. ${ }^{27}$ Moreover, environmental barriers, including poor social support, scheduling, cost (eg, parking), transportation, walking distance to the destination, time limits/attentional demands (eg, walk signals and crowds), ${ }^{8}$ physical barriers (eg, stairs and curbs) ${ }^{8}$ physical load, ${ }^{8}$ poor neighbourhood walkability (ie, traffic, difficult terrain and safety), ${ }^{26}$ lack of benches for resting and public bathrooms ${ }^{9}$ and inclement weather, ${ }^{6} 1011$ can limit outdoor walking for seniors.

Behavioural interventions designed to improve participation in outdoor walking should address the interaction between individual and environmental factors influencing outdoor community mobility. ${ }^{10}$ This interaction requires the capacity and the self-efficacy to manage eight dimensions of community mobility: distances, temporal factors, ambient conditions, physical load, terrain, attentional demands, postural transitions and traffic density. ${ }^{10}$ Although numerous studies have aimed to increase walking and physical activity in older adults, ${ }^{12-19}$ none of the interventions incorporated activities designed to build competency in the eight dimensions of community mobility. ${ }^{10}$ Select studies that required participants to practice community walking skills in contextually relevant environments are limited to people with stroke. ${ }^{20}$

Previous intervention research provides a basis for designing a complex intervention to improve outdoor walking. Task-oriented training is a therapeutic approach to improving outdoor walking skills that has been shown to improve walking self-efficacy in older adults, ${ }^{21}$ while a traditional approach focusing on impairments did not. ${ }^{22}$ Nordic pole walking may be a useful strategy to complement task-oriented outdoor walking training as it has been shown to improve functional capacity, gait speed, walking endurance and quality of life in healthy older adults and those with various health conditions. ${ }^{23-25}$ Social support from a walking partner or group is a strategy that can help adults to walk regularly. ${ }^{26}$ Walks in parks can facilitate socialisation and provide a regular change in surroundings. ${ }^{27}$ Tailored advice may be an important component given it has been as effective as supervised exercise or group walking in adults. ${ }^{28}$

In a meta-analysis of 19 trials of group walking, ${ }^{12}$ only seven focused on older adults aged $60+$ years. In five of these seven studies, walking groups were run in the community for 2-3 months with follow-up periods ranging from 3 months to 12 months. Effect sizes (ESs) were larger for follow-up periods greater than 6 months compared with those less than 6 months. This suggests that the duration of follow-up should extend beyond 6 months to adequately capture changes in physical activity behaviour. Self-report questionnaires were the most common measure of physical activity across studies. Although questionnaires can capture a range of physical activity behaviours, they have limitations in reliability and validity partially related to reliance on participant recall. ${ }^{29}$ Use of accelerometers with global positioning system (GPS) devices can address these limitations as they provide a direct and accurate measure of physical activity in older adults. ${ }^{30}$

We have completed a two-group, pilot randomised controlled trial (RCT) of a theory-informed outdoor mobility intervention among older adults who reported a limitation in outdoor community mobility. ${ }^{31}$ Quantitative and qualitative findings supported the safety and feasibility of the trial protocol and the potential of the intervention to improve confidence to walk outdoors, walking endurance, social participation and mental health. Evidence of effectiveness from a rigorously designed study is needed to justify widespread community implementation of this programme.

The primary objective of this study is to evaluate short-term and long-term effects of a 1-day educational workshop followed by a 10-week programme of group, task-oriented outdoor walking training (Getting Older Adults Outdoors [GO-OUT]) compared with the workshop and subsequent 10 weekly reminders (WR), on increasing outdoor walking activity in older adults with difficulty walking outdoors. A secondary objective is to evaluate the effect of GO-OUT on self-reported outdoor walking, physical activity, lifespace mobility, participation, emotional health, HRQL, balance, leg strength, walking self-efficacy, walking speed, walking endurance, heart rate (HR) and blood pressure (BP). As part of a planned process evaluation, we will explore participants' perceptions of intended and unintended short-term and longterm consequences of the interventions, intervention components considered as potent, mechanisms of effect, modifying influences of disability level, sex, neighbourhood walkability, weather, car access, study site and recommendations for future programme delivery models.

\section{METHODS AND ANALYSIS Trial design}

A two-group, evaluator-blinded, stratified, RCT with a qualitative component is being conducted (protocol version 1, 8 January 2019) in four Canadian cities (Edmonton [pop. 932 546], Winnipeg [pop. 705 244], Toronto [pop. 2731571 ] and Montreal [pop. 1704 694]). ${ }^{32}$ Data collection began in May 2018 and will continue until May 2020. The Framework for Enhancing the Value of Research for Dissemination and Implementation ${ }^{33}$ was used to guide the study design to optimise the application, replication and translation of results to the community setting. The Consolidated Standards of Reporting Trials statement will guide reporting on study results. ${ }^{34}$ The Standard Protocol Items: Recommendations for Interventional Trials statement was used to guide reporting of the protocol. ${ }^{35}$ Table 1 provides the timeline for enrolment, interventions and the administration of study measures.

\section{Eligibility criteria}

The target population for the trial is ambulatory older adults living in the community who have difficulty walking outdoors. 
Table 1 Schedule of enrolment, interventions and administration of study measures

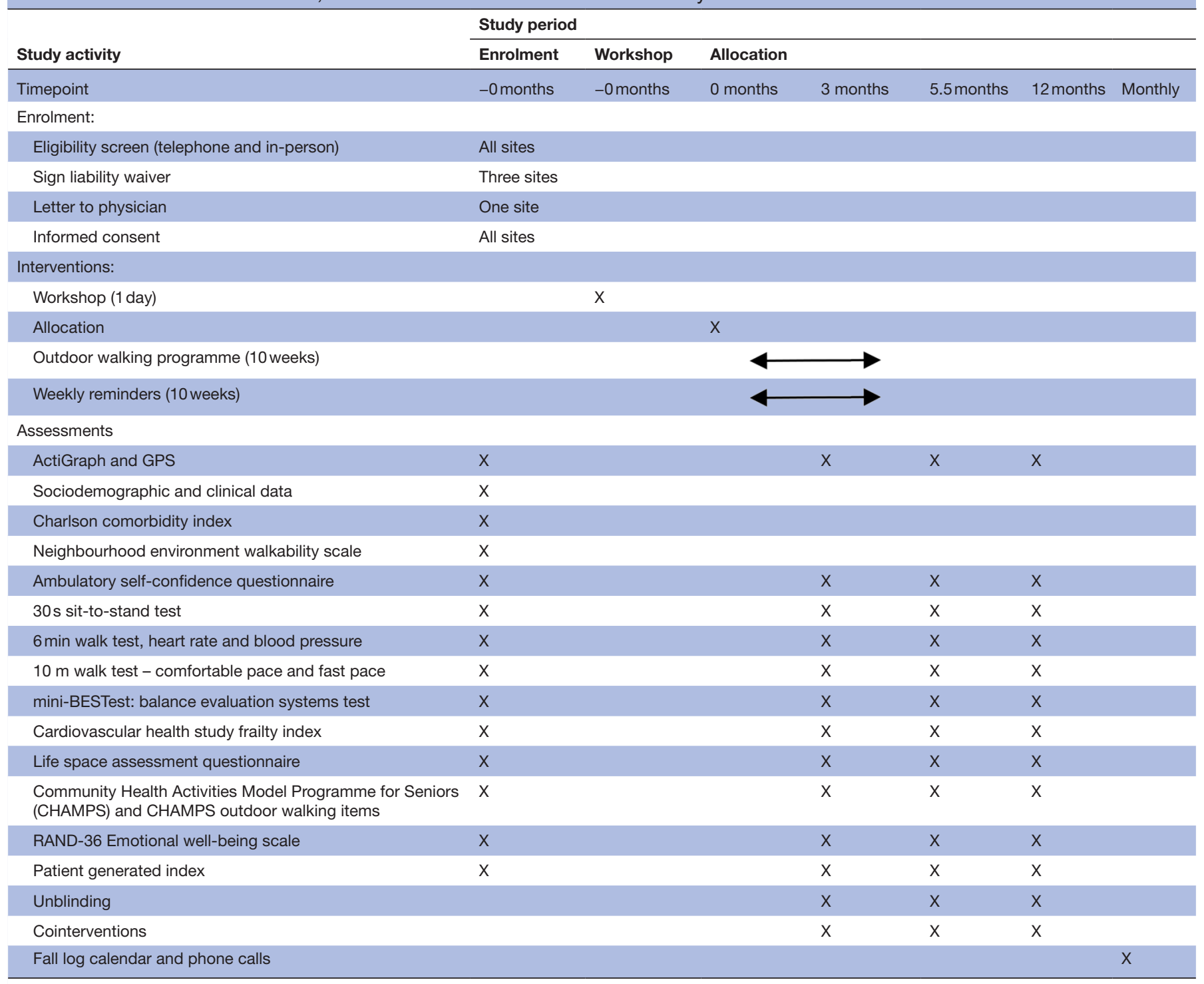

GPS, global positioning system; RAND, Research ANd Development.

\section{Inclusion criteria}

Individuals meeting the following inclusion criteria are considered eligible: (1) older adults defined as age 65 years or older; (2) living independently in the community; (3) ambulatory defined as self-reported ability to walk at least one block $(\sim 50 \mathrm{~m})$ continuously on a flat surface with or without a walking aid and without supervision; (4) self-reported difficulty walking in the outdoor community environment (this could include physical impairments or decreased confidence); (5) willingness to sign a liability waiver (required at three sites) or send a letter to their physician (required at one site) regarding clearance to exercise; (6) mental competency defined by a score of at least 18 out of 22 on the Mini-Mental State Exam telephone version ${ }^{36}$; (7) available for a scheduled workshop and at least 5 of
10 weeks of the outdoor walking programme; and (8) ability to speak and understand English.

\section{Exclusion criteria}

Individuals are excluded if they meet any of the following criteria: (1) physically active defined as self-reported participation in physical activities, such as walking and playing golf, 150 min per week; (2) currently receiving rehabilitation treatment, such as physical or occupational therapy, for goals related to walking (self-report); (3) at high falls risk defined by meeting one or more of the following American Geriatric Society criteria ${ }^{37}$ : (A) $\geq 2$ falls in the last 12 months or presents with an acute fall; (B) cardiac, respiratory, peripheral vascular or other health conditions that would prevent safe and full participation in the interventions (self-report); (C) postural hypotension defined as a drop in systolic BP of $>20 \mathrm{~mm} \mathrm{Hg}$ or a drop 


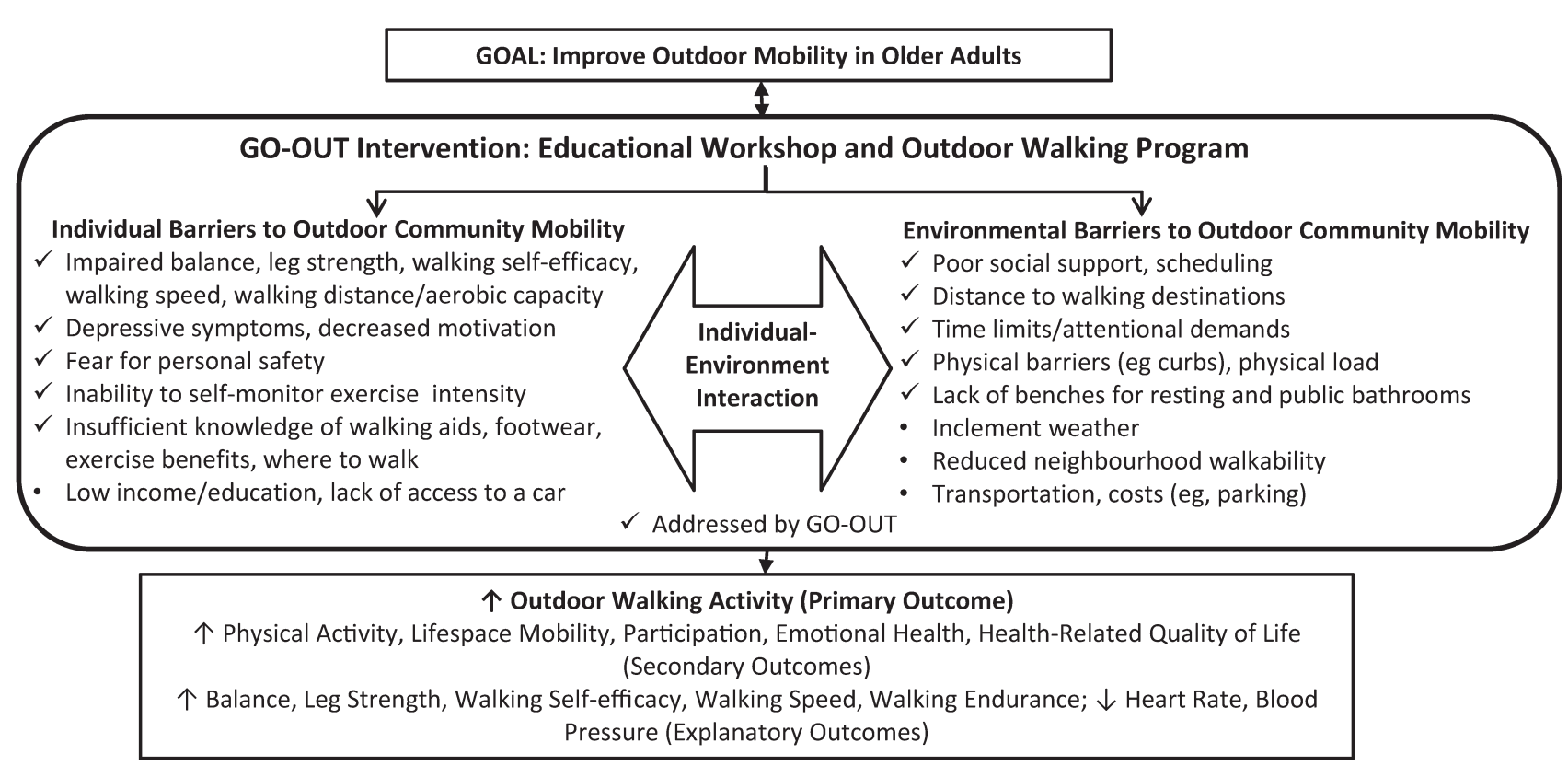

Figure 1 Conceptual framework for the GO-OUT intervention. GO-OUT, Getting Older Adults Outdoors.

in diastolic BP of $>10 \mathrm{~mm} \mathrm{Hg}$ taken after lying supine for 5 min and after standing for $2 \mathrm{~min}$; (D) resting $\mathrm{HR}<45$ or $>100$ beats per minute; and (E) severe limitation in visual acuity defined as self-reported difficulty reading the newspaper while wearing regular reading glasses or bifocals, or self-reported inability to distinguish a person's facial features from across a room while wearing glasses. ${ }^{38}$ Individuals excluded due to postural hypotension, severely limited visual acuity or abnormal resting HR are asked to see their family physician.

\section{Interventions}

Participants are randomised to one of two intervention groups: (1) workshop and outdoor walking programme (GO-OUT) and (2) workshop and weekly reminders (WR). Figure 1 presents the conceptual framework for the GO-OUT intervention developed based on current theory and research and modified based on pilot results. ${ }^{31}$

\section{Workshop}

Prior to randomisation, groups of up to 18 participants complete a 1-day educational workshop held at a convenient community location (eg, hospital or community centre). During the workshop, participants circulate in small groups of 2-3 to 8 interactive stations covering the following topics: Canadian physical activity guidelines for older adults ${ }^{39}$; setting SMART (ie, specific, measurable, achievable, realistic and timely) goals; pedometer use; Nordic pole walking; foot care, footwear, proper walking pattern; falls prevention; monitoring exercise intensity and safety; and postural awareness and balance exercises. Table 2 presents workshop station activities and the supporting rationale. Participants receive an educational workbook with content and activities for each topic and a pedometer. The process for teaching the workshop incorporates strategies to increase knowledge, skill, intention and self-efficacy ${ }^{40}$ to walk outdoors and cognitive strategies to help to prevent falls. The group nature of the workshop is expected to facilitate social connections and support. Each station is facilitated by a graduate student in a health professional programme or a health professional who has experience working with older adults and individuals with health conditions and holds cardiopulmonary resuscitation (CPR) certification.

\section{Outdoor walking programme}

Following completion of the workshop, participants in the experimental group participate in a progressive, task-specific group outdoor walking programme during good weather months (ideally June-August). The group consists of a maximum of nine participants and three facilitators to achieve a 3:1 ratio to optimise safety and formation of subgroups of participants by ability level. Groups may adjust the ratio depending on the level of supervision needed. The lead facilitator is a health professional with the expertise to modify the programme as needed and advise on appropriate use of ambulatory aids for individuals with chronic conditions, ability to encourage participation and CPR certification. The facilitator assistants (up to two per group) are health professionals or entry-to-practice/graduate students in health sciences-related programmes. Two 1-hour sessions are completed each week for 10 weeks. The same activities are completed during both sessions within the same week. Guidelines for cancelling sessions due to inclement weather are provided. Each session includes a $10 \mathrm{~min}$ warm-up, a distance walk, practice of a specific outdoor 
Table 2 Workshop station activities and rationale

\begin{tabular}{|c|c|c|}
\hline Station & Activities & Rationale \\
\hline $\begin{array}{l}\text { Station 1: Canadian Physical } \\
\text { Activity Guidelines for Older Adults } \\
65 \text { years and older }\end{array}$ & $\begin{array}{l}\text { 1. Review the Canadian Physical Activity Guidelines for Older } \\
\text { Adults and types of moderate- to vigorous-intensity aerobic } \\
\text { physical activities. } \\
\text { 2. Review a physical activity log and document minutes of } \\
\text { moderate- to vigorous-intensity aerobic physical activity in the } \\
\text { last week. }\end{array}$ & $\begin{array}{l}\text { Review of physical activity recommendations }{ }^{39} \text { is } \\
\text { expected to reinforce intervention credibility. Review } \\
\text { of benefits of following recommendations (outcome } \\
\text { expectations) is expected to motivate outdoor walking. }{ }^{40} \\
\text { Identifying types of moderate to vigorous intensity } \\
\text { aerobic physical activities is expected to help individuals } \\
\text { understand which activities would be associated with } \\
\text { health benefits. }\end{array}$ \\
\hline Station 2: setting SMART goals & $\begin{array}{l}\text { 1. Review how to set a SMART (specific, measurable, achievable, } \\
\text { realistic and timely) goal; write a SMART goal to improve } \\
\text { outdoor walking. } \\
\text { 2. Identify strategies to overcome three challenges to outdoor } \\
\text { walking (action plan). }\end{array}$ & $\begin{array}{l}\text { Setting personalised goals may promote outdoor } \\
\text { walking and participation based on research } \\
\text { poststroke }^{9495} \text { through creating intention or motivation for }^{\text {performing a certain behaviour. }}{ }^{96}\end{array}$ \\
\hline Station 3: pedometers & $\begin{array}{l}\text { 1. Review guidelines for daily step count. } \\
\text { 2. Review how to use a pedometer and practice while walking. } \\
\text { 3. Review a pedometer tracking log to record steps. }\end{array}$ & $\begin{array}{l}\text { Use of pedometers can be motivating, }{ }^{31} \text { and may promote } \\
\text { walking, }{ }^{97} \text { particularly when pedometer-based step goals } \\
\text { match physical activity guidelines. }{ }^{9899}\end{array}$ \\
\hline Station 4: Nordic pole walking & $\begin{array}{l}\text { 1. Review health benefits of Nordic pole walking. } \\
\text { 2. Review and practice how to adjust wrist straps and poles and } \\
\text { walk with them. }\end{array}$ & $\begin{array}{l}\text { Nordic pole walking programmes have been shown } \\
\text { to improve functional capacity, gait speed, walking } \\
\text { endurance and quality of life in healthy older adults and } \\
\text { those with various health conditions. }{ }^{23-25}\end{array}$ \\
\hline $\begin{array}{l}\text { Station } 5 \text { : foot care, footwear, and } \\
\text { proper walking pattern }\end{array}$ & $\begin{array}{l}\text { 1. Review of proper foot care and footwear and examine sample } \\
\text { shoes. } \\
\text { 2. Review video of a normal walking pattern and discuss } \\
\text { elements; video record each participant walking and review } \\
\text { the videos. }\end{array}$ & $\begin{array}{l}\text { Walking shoe guidelines }{ }^{100} \text { can help prevent falls in } \\
\text { older adults (www.preventfalls.ca). Walking with correct } \\
\text { posture and gait pattern can help to decrease energy } \\
\text { expenditure. }\end{array}$ \\
\hline Station 6: falls prevention & $\begin{array}{l}\text { 1. Review the cognitive strategy of 'if-then' plans to decrease } \\
\text { risk of falls. } \\
\text { 2. Complete the Take Action to Prevent Falls Check-Up } \\
\text { questionnaire and review recommendations for different falls } \\
\text { risk factors (including a home safety checklist); write one } \\
\text { SMART goal to reduce falls risks. }\end{array}$ & $\begin{array}{l}\text { 'If-then' statements are a cognitive strategy used } \\
\text { to develop intentions to prevent falls while walking } \\
\text { outside. Intentions are considered a main predictor of } \\
\text { behaviour. }{ }^{96101}\end{array}$ \\
\hline $\begin{array}{l}\text { Station } 7 \text { : monitoring exercise } \\
\text { intensity and safety }\end{array}$ & $\begin{array}{l}\text { 1. Review the modified Borg scale } \mathrm{e}^{102103} \text { and the level of } \\
\text { perceived exertion (moderate-somewhat hard) that is } \\
\text { considered moderate-intensity aerobic exercise. } \\
\text { 2. Review warning signs and symptoms to stop exercising. } \\
\text { 3. Practice rating your level of perceived exertion after a } 5 \text { min } \\
\text { brisk walk. }\end{array}$ & $\begin{array}{l}\text { Knowledge of the signs and symptoms signalling an } \\
\text { adverse response to exercise is expected to enable self- } \\
\text { monitoring of exercise intensity to identify the need to rest. }\end{array}$ \\
\hline $\begin{array}{l}\text { Station 8: postural awareness and } \\
\text { balance exercises }\end{array}$ & $\begin{array}{l}\text { Review and practice five home exercises to improve balance } \\
\text { and lower extremity strength; review the need for handholds } \\
\text { and supportive footwear when performing exercises for safety. } \\
\text { Exercises include }^{104} \text { : } \\
\text { 1. Walk stance. } \\
\text { 2. Hip abduction in standing. } \\
\text { 3. Heel raises. } \\
\text { 4. Hip flexion in standing. } \\
\text { 5. Sit-to-stand. }\end{array}$ & $\begin{array}{l}\text { The Canadian Society for Exercise Physiology }{ }^{39} \text { and the } \\
\text { American College of Sports Medicine }{ }^{105} \text { recommend that } \\
\text { older adults perform physical activities to increase balance } \\
\text { and reduce falls risk. There is substantial evidence that } \\
\text { physical activity reduces risk of falls and injuries from falls, } \\
\text { prevents or mitigates functional limitations and is effective } \\
\text { therapy for people with many chronic diseases. }{ }^{105}\end{array}$ \\
\hline Proper use of walking aids & $\begin{array}{l}\text { During a workshop break, a physical therapist evaluates the height } \\
\text { and use of walking aids with each individual user and makes } \\
\text { adjustments and recommendations as appropriate. }\end{array}$ & $\begin{array}{l}\text { Ensuring the appropriate fit of mobility devices for } \\
\text { participants is a strategy to optimise safe outdoor } \\
\text { mobility. }{ }^{94}\end{array}$ \\
\hline
\end{tabular}

walking skill, a distance walk and a $10 \mathrm{~min}$ cool down. The warm up and cool down include stretching, functional strengthening exercises and balance exercises taught during the workshop.

The outdoor walking programme is theory based. Walking activities each week are designed to build competency in two to four of the eight dimensions of outdoor ambulation as shown in table $3{ }^{10}$ The programme incorporates the principles of task-specific training by emphasising repetitive practice of progressively more difficult outdoor walking tasks. The programme incorporates progression of difficulty by: (1) providing two different walking distance goals each session for people with gait speeds $<$ and $\geq 0.8 \mathrm{~m} / \mathrm{s}$; (2) progressively increasing walking distance goals, and the variety and challenge of walking activities (eg, walking farther, carrying objects and navigating slopes/uneven surfaces) over the 10-week period; and (3) allowing the lead facilitator to adjust the level of difficulty each session to ensure participants are challenged. The outdoor walking programme incorporates strategies for increasing self-efficacy to walk outdoors by: (1) enabling successful practice of outdoor walking tasks in contextually relevant environments (performance accomplishments); (2) using a qualified lead facilitator who can provide credible feedback on task performance (verbal persuasion); (3) providing opportunities to observe successful task performance in peers with similar levels of challenge (vicarious experience); and (4) providing opportunities to practice outdoor walking tasks in a comfortable, safe and supervised environment (emotional arousal). Finally, the outdoor walking programme is conducted in one or 
Table 3 Outdoor walking programme activities

\begin{tabular}{|c|c|c|c|c|c|c|c|c|}
\hline \multirow[b]{2}{*}{ Week } & \multirow[b]{2}{*}{ Activities } & \multicolumn{7}{|c|}{ Community ambulation dimension targeted } \\
\hline & & D & PT & $\mathbf{T}$ & TF & PL & AD & TD \\
\hline 1 & $\begin{array}{l}\text { Distance walk: GS }<0.8 \mathrm{~m} / \mathrm{s}: 200 \mathrm{~m} \text {; GS } \geq 0.8 \mathrm{~m} / \mathrm{s}: 400 \mathrm{~m} \text {. } \\
\text { On level walking surface, for example, paved path: } \\
\text { Walking and turning. } \\
\text { Stepping sideways. } \\
\text { Starting and stopping. } \\
\text { Timed Up and Go - as an activity. } \\
\text { Distance walk: GS }<0.8 \mathrm{~m} / \mathrm{s}: 200 \mathrm{~m} \text {; GS } \geq 0.8 \mathrm{~m} / \mathrm{s}: 400 \mathrm{~m} \text {. } \\
\text { Review if-then statements to prevent falls. }\end{array}$ & $x$ & $\mathrm{x}$ & & & & & \\
\hline 2 & $\begin{array}{l}\text { Review of use of Nordic poles. } \\
\text { Distance walk: GS }<0.8 \mathrm{~m} / \mathrm{s}: 200 \mathrm{~m} \text {; GS } \geq 0.8 \mathrm{~m} / \mathrm{s}: 400 \mathrm{~m} \text {. } \\
\text { Walking on hills, curbs and stairs. } \\
\text { On challenging terrain, for example, grass: } \\
\text { Walking and turning. } \\
\text { Stepping sideways. } \\
\text { Starting and stopping. } \\
\text { Distance walk: GS }<0.8 \mathrm{~m} / \mathrm{s}: 200 \mathrm{~m} \text {; GS } \geq 0.8 \mathrm{~m} / \mathrm{s}: 400 \mathrm{~m} \text {. } \\
\text { Review if-then statements to prevent falls. }\end{array}$ & $\mathrm{X}$ & $x$ & $\mathrm{x}$ & & & & \\
\hline
\end{tabular}

Distance walk: GS $<0.8 \mathrm{~m} / \mathrm{s}: 225 \mathrm{~m}$; GS $\geq 0.8 \mathrm{~m} / \mathrm{s}: 425 \mathrm{~m}$ (walk around loop
track if possible and safe).
On paved path: 'crossing the street'.
On paved path: walking with a sudden stop.
Distance walk: GS $<0.8 \mathrm{~m} / \mathrm{s}: 225 \mathrm{~m}$; GS $\geq 0.8 \mathrm{~m} / \mathrm{s}: 425 \mathrm{~m}$.
Review if-then statements to prevent falls.
Distance walk: GS $<0.8 \mathrm{~m} / \mathrm{s}: 250 \mathrm{~m}$; GS $\geq 0.8 \mathrm{~m} / \mathrm{s}: 450 \mathrm{~m}$.
4

On various types of terrain: relay race.

Distance walk: GS $<0.8 \mathrm{~m} / \mathrm{s}: 250 \mathrm{~m}$; GS $\geq 0.8 \mathrm{~m} / \mathrm{s}: 450 \mathrm{~m}$.

Review if-then statements to prevent falls.

\begin{tabular}{|c|c|c|c|c|c|c|c|c|}
\hline 5 & $\begin{array}{l}\text { Review of use of Nordic poles. } \\
\text { Distance walk: GS }<0.8 \mathrm{~m} / \mathrm{s}: 300 \mathrm{~m} \text {; GS } \geq 0.8 \mathrm{~m} / \mathrm{s}: 500 \mathrm{~m} \text {. } \\
\text { On paved path: Nordic pole walking. } \\
\text { Distance walk: GS }<0.8 \mathrm{~m} / \mathrm{s}: 300 \mathrm{~m} \text {; GS } \geq 0.8 \mathrm{~m} / \mathrm{s}: 500 \mathrm{~m} \text {. } \\
\text { Review if-then statements to prevent falls. }\end{array}$ & $x$ & & & & & $x$ & \\
\hline 6 & $\begin{array}{l}\text { Distance walk: GS }<0.8 \mathrm{~m} / \mathrm{s}: 300 \mathrm{~m} \text {; GS } \geq 0.8 \mathrm{~m} / \mathrm{s}: 500 \mathrm{~m} \text {. } \\
\text { On paved path: 'crossing the street'. } \\
\text { On paved path: walking with a sudden stop. } \\
\text { On paved path: the above activities while carrying a load. } \\
\text { Distance walk: GS }<0.8 \mathrm{~m} / \mathrm{s}: 300 \mathrm{~m} \text {; GS } \geq 0.8 \mathrm{~m} / \mathrm{s}: 500 \mathrm{~m} \text {. } \\
\text { Review if-then statements to prevent falls. }\end{array}$ & $x$ & $x$ & & $X$ & $x$ & & \\
\hline 7 & $\begin{array}{l}\text { Walking on a nature path (using Nordic poles): } \mathrm{GS}<0.8 \mathrm{~m} / \mathrm{s} \text { : } 800 \mathrm{~m} \text { to } 1 \mathrm{~km} \text {; } \\
\mathrm{GS} \geq 0.8 \mathrm{~m} / \mathrm{s}: 1.5-2 \mathrm{~km} \text {. } \\
\text { 1. Visual screening of the route ahead and obstacle avoidance. } \\
\text { 2. Nordic pole walking. } \\
\text { 3. Participants are encouraged to talk with each other during the walk. } \\
\text { Review if-then statements to prevent falls. }\end{array}$ & $x$ & & $x$ & & & $x$ & \\
\hline 9 & $\begin{array}{l}\text { Distance walk: GS }<0.8 \mathrm{~m} / \mathrm{s}: 400 \mathrm{~m} \text {; GS } \geq 0.8 \mathrm{~m} / \mathrm{s}: 600 \mathrm{~m} \text { (walk around loop } \\
\text { track if possible and safe). } \\
\text { On paved path: 'crossing the street'. } \\
\text { On paved path: 'crossing the street', walking through a crowd. } \\
\text { Distance walk: GS }<0.8 \mathrm{~m} / \mathrm{s}: 400 \mathrm{~m} \text {; GS } \geq 0.8 \mathrm{~m} / \mathrm{s}: 600 \mathrm{~m} \text { (walk around loop } \\
\text { track if possible and safe). } \\
\text { Review if-then statements to prevent falls }\end{array}$ & $x$ & & & $x$ & & & $x$ \\
\hline 10 & $\begin{array}{l}\text { Distance walk: GS }<0.8 \mathrm{~m} / \mathrm{s}: 400 \mathrm{~m} \text {; GS } \geq 0.8 \mathrm{~m} / \mathrm{s}: 600 \mathrm{~m} \text {. } \\
\text { On paved path: 'carrying load'. } \\
\text { On paved path: 'carrying load', walking through a crowd. } \\
\text { Distance walk: GS }<0.8 \mathrm{~m} / \mathrm{s}: 400 \mathrm{~m} \text {; GS } \geq 0.8 \mathrm{~m} / \mathrm{s}: 600 \mathrm{~m} \text {. } \\
\text { Review if-then statements to prevent falls. }\end{array}$ & $x$ & & & & $x$ & & $x$ \\
\hline
\end{tabular}

$\mathrm{AD}$, attentional demands; D, distance; GS, gait speed; m, metres.; PL, physical load; PT, postural transitions; T, terrain; TD, traffic density; TF, temporal factors. 
more large park settings given the mental health benefits of exercising in a natural environment. ${ }^{41}$ Parks meeting the following criteria were selected based on preferences of older adults: terrains required for the weekly walking skills training; accessible by public and adapted transport; sufficient benches, washroom and parking facilities; attractive surroundings; and presence of other walkers (ie, not isolated). ${ }^{31}$

\section{Weekly reminders}

Participants in the WR group receive weekly reminders by telephone from the study coordinator for 10 weeks following completion of the workshop. Each weekly reminder involves scripted questions designed to reinforce information and skills taught at each workshop station and provide reminders to get physically active as outlined in table 4. Seven reminders include review of the 'if-then' cognitive strategy to prevent falls. Email scripts were prepared to send if the participant could not be reached by phone. The weekly reminders intervention was designed to equalise control for attention provided to participants in each group. The workshop plus reminders intervention was considered a lower cost, feasible alternative to the GO-OUT intervention given results from previous research suggesting tailored advice is as effective as walking groups for improving physical activity. ${ }^{28}$

\section{Standardisation of the interventions}

The workshop, weekly reminders and outdoor walking programme are standardised to facilitate consistent implementation across sites. A workshop facilitator guide outlines instructions and scripts for each station. Facilitators at each site undergo training for the workshop led by NMS or RB. The workshop training session is also attended by outdoor walking programme facilitators and involves review and practice of assigned stations. A weekly reminder facilitator guide outlines instructions and scripts for delivering each reminder. The outdoor walking programme facilitator guide outlines criteria for cancelling a session due to weather, roles of the leader and assistants, safety recommendations and the exercise protocol for required equipment, weekly activities, warm-up and cool-down. NMS and RB complete site visits to guide study implementation.

\section{Evaluations}

Trained evaluators blinded to intervention assignment complete in-person evaluations at 0 (baseline), 3, 5.5 and 12 months at community or academic centres. The time point of 5.5 months was chosen to enable evaluation of short-term effects of the interventions prior to the onset of cold weather. Study measures with established reliability and validity are administered according to standardised procedures outlined in a manual with links to training videos. The central site conducts an orientation session for site coordinators to review the evaluation manual and procedures. Site coordinators complete a training session with evaluators involving a review of the manual and practice administering the tests and questionnaires required for evaluations. To maintain blinding, participants are reminded not to reveal their study group during evaluations. To evaluate blinding, evaluators are asked to document if unblinding occurs and the intervention assignment at each follow-up evaluation. To optimise attendance at evaluations, participants are provided with a gift card.

\section{Data collection}

\section{Primary outcome measure of outdoor walking activity}

The primary study outcome of outdoor walking activity is being measured as the number of minutes per week spent in outdoor walking by synchronising walking data captured by accelerometry (ActiGraph GT3X+ activitymonitor [ActiGraph, Pensacola, Florida, USA]) with outdoor location and speed data measured by a GPS monitor (Qstarz BT-Q1000XT A-GPS Travel Recorder). The GT3X+ monitor includes a triaxial accelerometer to detect steps and activity counts. The reliability ${ }^{42}$ and validity of the GT3X+for measuring physical activity under both laboratory ${ }^{43}$ and free-living conditions ${ }^{44}$ has been established. The Qstarz A-GPS Travel Recorder was chosen for ease of use and capacity to accurately record GPS data (within $3 \mathrm{~m}$ ) for 5-40 days depending on the frequency of data capture. ${ }^{45}$ At each evaluation, participants are given an activity monitor and a GPS device and asked to wear both monitors over the right hip on a belt around the waist during waking hours for eight consecutive days. This wear period was chosen to minimise social desirability bias and confounding by day of the week on the amount of activity recorded. Participants are also asked to record when they put on and remove the monitors each day and when they go outdoors and come indoors on log sheets to help align the activity monitor and GPS data. We define a valid day as a minimum wear time of 10 hours per day and data from at least four valid days ${ }^{46}$ to estimate outdoor walking activity and secondary outcomes of physical activity. The number of $\geq 5$ min lifestyle bouts ${ }^{47}$ ( $>760$ counts per $\min$ ) and the number of $\geq 5 \mathrm{~min}$ purposeful walking bouts ${ }^{48}$ (intensity defined as $\geq 40$ steps per min) spent in outdoor walking over the number of wear days will be transposed to minutes spent in outdoor walking per week.

\section{Secondary outcome measures Walking endurance, $H R$ and $B P$}

The 6 min walk test ${ }^{49}$ is used to evaluate walking endurance. Participants are screened for relative and absolute contraindications to administering the 6 min walk test, ${ }^{49}$ which necessitate taking HR and BP after the participant has been resting in sitting for at least 5 min with back supported and feet flat on the floor after sitting in an environment free from distractions. HR is taken through manual palpation of the radial artery for $1 \mathrm{~min} .{ }^{50} \mathrm{BP}$ is taken using a manual or automated cuff. Resting HR and BP are documented. Relative contraindications include, but are not limited to, resting HR $>120 \mathrm{bpm}$ (tachycardia); HR $<60 \mathrm{bpm}$ (bradycardia); resting systolic $\mathrm{BP} \geq 160 \mathrm{~mm} \mathrm{Hg}$ (stage 2 hypertension) and resting diastolic $\mathrm{BP}>100 \mathrm{~mm} \mathrm{Hg}$ (stage 2 hypertension). On passing the screen, participants are asked to walk unassisted using their usual mobility device 


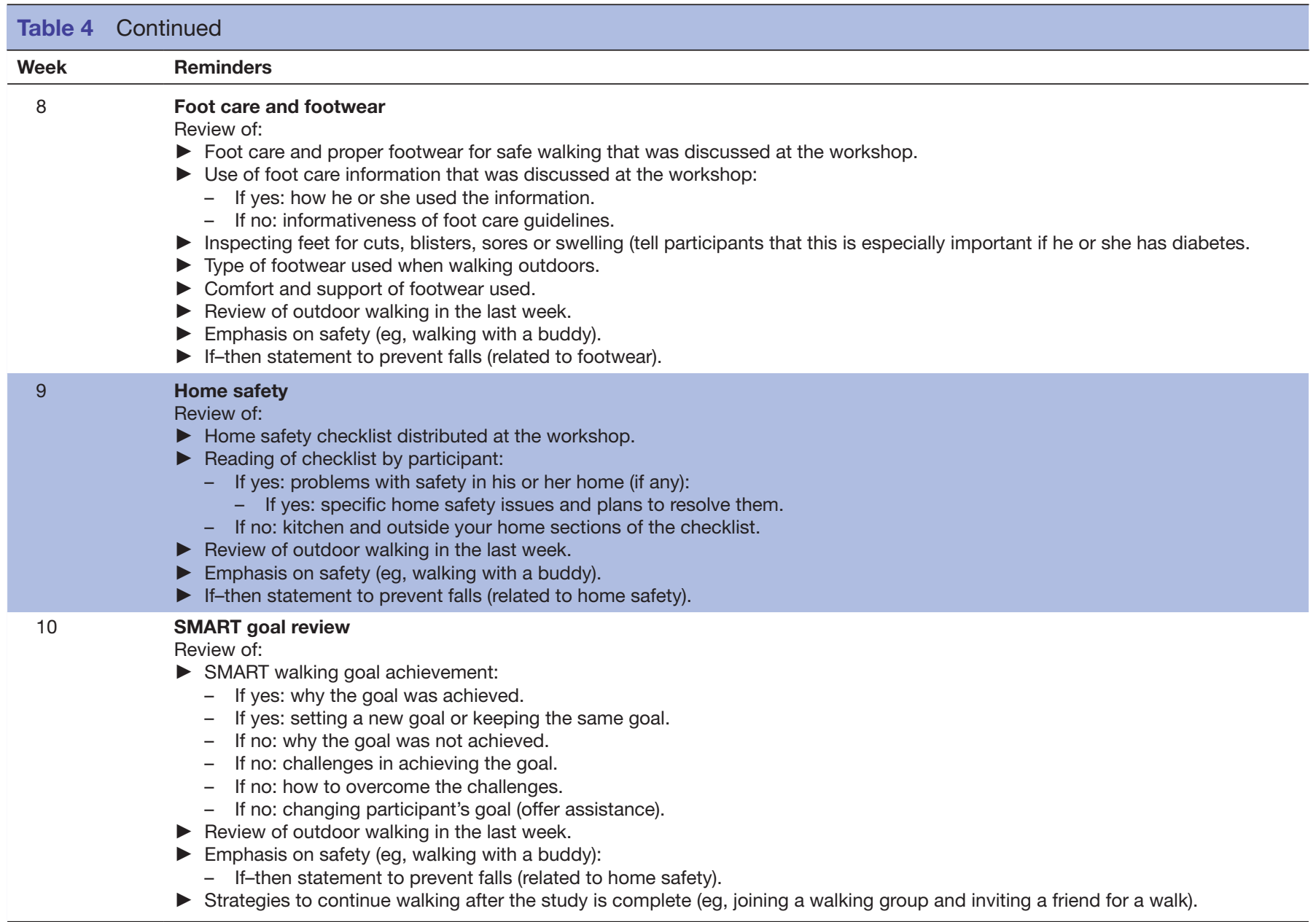

SMART, specific, measurable, achievable, realistic and timely.

back and forth along a straight, $30 \mathrm{~m}$ walkway. The participant is asked to use his or her usual assistive devices and corrective eyewear. Standardised encouragement phrases are provided each minute. The distance walked in metres in $6 \mathrm{~min}$ is documented. Test-retest reliability of the $6 \mathrm{~min}$ walk test is excellent in older adults with and without disability as indicated by intraclass correlation coefficients (ICCs) ranging from 0.95 to 0.99 .5152

\section{Balance}

The 14-item Mini Balance Evaluation Systems test ${ }^{53}$ (miniBESTest) is used to assess balance capacity. Item scores are summed to yield a total score that can range from 0 to 28 points. ${ }^{54}$ Higher scores indicate better balance. The ICC estimate for inter-rater reliability is 0.91 , and there is evidence of construct validity. ${ }^{5355}$

\section{Lower extremity strength}

Lower extremity strength is evaluated indirectly using the sit-to-stand test, ${ }^{567}$ scored as the number of sit-to-stands completed in $30 \mathrm{~s}$. The ICC estimate for test-retest reliability is $0.89 .^{56}$

\section{Ambulation self-efficacy}

The 22-item ambulatory self-confidence questionnaire ${ }^{58}$ is used to evaluate self-efficacy to walk at home and/or in the community. The total score is the mean of itemlevel scores and can range from 0 to 10 . The ICC estimate for test-retest reliability is $0.92(95 \%$ CI 0.87 to 0.95$){ }^{58}$ There is evidence supporting construct validity. ${ }^{58}$

\section{Walking speed, comfortable and fast pace}

The $10 \mathrm{~m}$ walk test ${ }^{59}$ is used to measure walking speed at a comfortable and fast pace over the middle $10 \mathrm{~m}$ portion of a $14 \mathrm{~m}$ walkway. No practice trial is performed. The participant is asked to use his or her usual assistive device(s) and corrective eyewear. Test-retest reliability is excellent in older adults with and without disability as indicated by ICC values between 0.90 and 0.98 for tests conducted at normal and maximal paces. ${ }^{60-62}$

\section{Life space mobility}

The life space assessment questionnaire ${ }^{63}$ is used to obtain a composite score of the level, degree of independence and frequency of attainment of mobility in five living spaces in the past 4 weeks: rooms in the house; the area outside the house; places in the neighbourhood; places outside the neighbourhood but within the city; and places outside the city. Item scores are summed to yield a total score that can range from 0 (totally bed bound) to 120 (travels out of the city every day without assistance). ${ }^{64}$ 
The questionnaire demonstrates excellent test-retest reliability in older adults (ICC estimate $=0.96,95 \%$ CI 0.95 to 0.97) ${ }^{63}$ Construct validity is supported. ${ }^{63}$

\section{Participation, physical activity and outdoor walking}

The self-reported Community Health Activities Model Program for Seniors ${ }^{65}$ (CHAMPS) questionnaire, validated in older adults, is used to evaluate participation and physical activity. To complete the CHAMPS, hours spent performing 40 social, leisure and physical activities in a typical week during the last 4 weeks is indicated. Specific items are combined to produce subscales for time spent in moderate- to vigorous-intensity aerobic physical activity, walking and total participation time per week. Activities with an assigned metabolic equivalent value $\geq 3.0$ are used to estimate moderate- to vigorous-intensity aerobic physical activity. We asked participants to complete a second set of the walking items on the CHAMPS that were reworded to indicate the activity is outdoors.

\section{Health-related quality of life}

The Research ANd Development-36 $6^{66}$ (RAND-36) and the Patient Generated Index ${ }^{6768}$ (PGI) are used to evaluate HRQL. The RAND-36 is a 36 -item generic self-report measure designed to measure eight health concepts: physical functioning, role limitations caused by physical health problems, role limitations caused by emotional problems, social functioning, emotional well-being, energy/fatigue, pain and general health perceptions. We are using the emotional well-being scale to measure emotional health. It is scored from 0 to 100 where higher scores represent the most optimal health state. To complete the PGI, individuals are asked to list the five most important areas of their life that are affected by their health. A sixth box is provided to represent all other areas of their life not previously mentioned. Next, they are asked to rate the degree to which these areas are affected on a scale from 0 to 10 , where 0 is the worst level imaginable and 10 represents the best level of function possible. Lastly, participants are given 12 imaginary points to 'spend' on their chosen areas to represent the weight or importance of each area. The score on the $0-10$ scale and the weighting are multiplied for each area chosen. The PGI has been shown to be reliable for comparisons between groups and to demonstrate good validity. ${ }^{68}$

\section{Frailty}

The Cardiovascular Health Study Frailty Index ${ }^{69}$ is used to evaluate the presence of five frailty indicators: (1) unintentional weight loss in the last year (self-report); (2) exhaustion (self-report); and (3) low physical activity (self-report), slow walking speed and weak grip strength on the dominant side measured by dynamometer using reported operational definitions. ${ }^{69}$ Level of frailty is classified based on the number of indicators present: frail (3-5 indicators present); prefrail (1-2 indicators present); and not frail (no indicators present) ${ }^{69}$

\section{Neighbourhood walkability}

The Neighbourhood Environment Walkability Scale ${ }^{70} 71$ (NEWS) assesses how participants perceive the environmental features of their neighbourhood that relate to walkability using ordinal scales. The NEWS has 13 sections with specific questions inquiring about the types of buildings, accessibility to different businesses and services, infrastructure for pedestrians, cyclists and motorists, neighbourhood cleanliness and aesthetics, and safety. Scoring for the confirmatory factor analysis version of the NEWS produces eight multifactor items and five single factor items. Higher scores indicate higher walkability for all subscales. Evidence of factorial and criterion validity has been reported. ${ }^{71} 72$

\section{Comorbidity, body mass index and sociodemographic characteristics}

At baseline, we are collecting data on comorbidity using the Charlson comorbidity index ${ }^{73}$ (CCI). The CCI has been shown to have moderate to good inter-rater reliability and content validity. ${ }^{75}$ We are also taking a measurement of height and asking participants to self-report their weight (to compute body mass index), age, sex, education, income, smoking status, use of glasses and/or mobility devices, social support, car access, medications and reasons for outdoor walking limitation.

\section{Cointerventions}

At each evaluation, data on participation in cointerventions (eg, physical therapy) that may influence walking activity are collected.

\section{Adverse events}

Adverse events occurring during scheduled study sessions (ie, evaluations, workshop and outdoor walking group) are documented using a standardised form. In this study, a fall is being defined as 'an unexpected event in which the participant comes to rest on the ground, floor, or lower level'. ${ }^{76}$ Falls are being classified as non-injurious and injurious. An injurious fall is one that results in an injury requiring medical care or hospitalisation. ${ }^{77}$ Falls that occur outside study sessions are tracked using a 'gold standard approach'. ${ }^{76}$ Participants are provided with monthly falls log calendars and asked to record falls occurrence throughout the study. The study coordinator calls people monthly to obtain information on falls occurrence and any resulting injuries. This approach to monitoring adverse events, including falls, was feasible to implement during our pilot trial. ${ }^{31}$

\section{Quantitative process evaluation}

Our process evaluation was designed to capture two main aspects: delivery (implementation fidelity) and receipt of (level of participant engagement with) the intervention. ${ }^{78}$ Table 5 describes the site-level and participant-level process indicators used to evaluate these aspects. The evaluation of implementation fidelity involves completion of checklists on provision of intervention components. The evaluation of participant engagement involves 
Table 5 Process evaluation measures at the site level and participant level

\begin{tabular}{|c|c|c|c|c|c|}
\hline Level & Process variable & Indicator & Response scale & Denominator & Findings to be reported \\
\hline \multirow[t]{2}{*}{ Site } & Workshop training. & $\begin{array}{l}\text { Workshop facilitators participated } \\
\text { in a workshop training session } \\
\text { that involved practice of assigned } \\
\text { stations. }\end{array}$ & Yes/no. & Total \# of sites. & $\begin{array}{l}\mathrm{n}(\%) \text { of sites } \\
\text { implementing training. }\end{array}$ \\
\hline & $\begin{array}{l}\text { Workshop } \\
\text { implementation fidelity. }\end{array}$ & $\begin{array}{l}\text { Implementation of stations } 1-8 \text { as } \\
\text { described in the GO-OUT Workshop } \\
\text { Facilitator Guide. }\end{array}$ & Yes/no. & $\begin{array}{l}\text { Total \# of workshops } \\
\text { completed. }\end{array}$ & $\begin{array}{l}\mathrm{n}(\%) \text { of times each } \\
\text { station implemented for } \\
\text { eight stations within and } \\
\text { across sites. }\end{array}$ \\
\hline
\end{tabular}

\begin{tabular}{|c|c|c|c|c|c|}
\hline & & $\begin{array}{l}\text { Level of walking activity achieved } \\
\text { evaluated using pedometer. }\end{array}$ & $\begin{array}{l}\text { Number of steps taken } \\
\text { during workshop. }\end{array}$ & N/A & $\begin{array}{l}\text { Mean, SD and range of } \\
\text { steps taken within and } \\
\text { across sites. }\end{array}$ \\
\hline & $\begin{array}{l}\text { Outdoor walk group } \\
\text { implementation fidelity. }\end{array}$ & $\begin{array}{l}\text { Implementation of } 10 \text { weekly session } \\
\text { components (warm-up, first walk, } \\
\text { weekly activity, second walk and } \\
\text { cool-down) as described in the GO- } \\
\text { OUT Outdoor Walk Group Facilitator } \\
\text { Guide. }\end{array}$ & $\begin{array}{l}\text { Yes/no. } \\
\text { Reflective notes written } \\
\text { by walk group leader. }\end{array}$ & $\begin{array}{l}\text { Total \# of week } 1 \\
\text { sessions; total \# of week } \\
2 \text { sessions; ... total \# of } \\
\text { week } 10 \text { sessions. }\end{array}$ & $\begin{array}{l}\mathrm{n}(\%) \text { of times each } \\
\text { component implemented } \\
\text { (ie, warm-up, first walk, } \\
\text { weekly activity, second } \\
\text { walk and cool-down) } \\
\text { for } 10 \text { weeks within and } \\
\text { across sites. }\end{array}$ \\
\hline & $\begin{array}{l}\text { Weekly reminders } \\
\text { implementation fidelity. }\end{array}$ & $\begin{array}{l}\text { Implementation of } 10 \text { weekly } \\
\text { reminder components (use } \\
\text { of booklet during the call and } \\
\text { adherence to the script) as } \\
\text { described in the GO-OUT Weekly } \\
\text { Reminders Facilitator Guide. }\end{array}$ & $\begin{array}{l}\text { Yes/no. } \\
\text { Reflective notes written } \\
\text { by coordinator. }\end{array}$ & $\begin{array}{l}\text { Total \# of participants in } \\
\text { control group. }\end{array}$ & $\begin{array}{l}\mathrm{n}(\%) \text { of times each } \\
\text { component implemented } \\
\text { (ie, use of booklet and } \\
\text { script) per week for } \\
10 \text { weeks within and } \\
\text { across sites. }\end{array}$ \\
\hline \multirow{4}{*}{ Participant } & & Completion of stations $1-8$. & Yes/no for each station. & $\begin{array}{l}\text { \# of randomised } \\
\text { participants in each } \\
\text { group. }\end{array}$ & $\begin{array}{l}\mathrm{n}(\%) \text { of randomised } \\
\text { participants completing } \\
\text { each station by group. }\end{array}$ \\
\hline & $\begin{array}{l}\text { Engagement with } \\
\text { outdoor walk group. }\end{array}$ & Attendance at each session. & $\begin{array}{l}\text { Yes/no converted to \% } \\
\text { of total \# of sessions } \\
\text { attended. }\end{array}$ & $\begin{array}{l}\text { Total \# of participants } \\
\text { randomised to outdoor } \\
\text { walk group. }\end{array}$ & $\begin{array}{l}n(\%) \text { attending: } \\
0 \%-25 \% \\
25 \%-50 \% \\
50 \%-75 \% \\
75 \%-100 \% \text { of sessions. }\end{array}$ \\
\hline & & $\begin{array}{l}\text { Use of Nordic walking poles each } \\
\text { session. }\end{array}$ & $\begin{array}{l}\text { Yes/no converted to \% of } \\
\text { total \# of sessions. }\end{array}$ & $\begin{array}{l}\text { Total \# of participants } \\
\text { randomised to outdoor } \\
\text { walk group. }\end{array}$ & $\begin{array}{l}\text { n (\%) using poles in: } \\
0 \%-25 \% \\
25 \%-50 \% \\
50 \%-75 \% \\
75 \%-100 \% \text { of sessions. }\end{array}$ \\
\hline & $\begin{array}{l}\text { Engagement with } \\
\text { weekly phone } \\
\text { reminders. }\end{array}$ & $\begin{array}{l}\text { Receipt of each of } 10 \text { weekly } \\
\text { reminders and method (email, } \\
\text { phone and both). }\end{array}$ & Yes/no. & $\begin{array}{l}\text { Total \# of participants } \\
\text { randomised to control } \\
\text { group. }\end{array}$ & $\begin{array}{l}\mathrm{n}(\%) \text { receiving reminder } \\
1,2,3, \ldots 10 .\end{array}$ \\
\hline
\end{tabular}

GO-OUT, Getting Older Adults Outdoors.

documenting attendance at the workshop and outdoor walking sessions and study withdrawals.

\section{Qualitative process evaluation}

In alignment with recommended approaches for evaluating complex interventions, ${ }^{79} 80$ qualitative methods, specifically a qualitative descriptive approach, ${ }^{81}$ will be used to obtain an in-depth understanding of participants' experiences with the study interventions as well as participants' recommendations for alternate programme models (eg, GO-OUT for winter months) to guide future research. Within the framework of evaluating the complex GO-OUT interventions, we are specifically interested in understanding the intended and unintended 
consequences of the interventions, potent intervention components, mechanisms of effect and potential influences of effect modifiers (eg, disability level, sex, neighbourhood walkability, weather, car access and study site) as experienced by the participants. More broadly, we are also interested in understanding the meaning participants attach to their experiences of participating in the workshop, outdoor walking group and reminder intervention components. A maximum of four participants from each intervention group per site (total $n=32$ ) will be invited to participate in telephone interviews with a trained interviewer. Site coordinators will selectively contact participants to achieve a balance on two variables: walking deficit (gait speed $<0.8 \mathrm{~m} / \mathrm{s}$ vs $\geq 0.8 \mathrm{~m} / \mathrm{s}$ ) and sex. Semistructured interviews lasting approximately 60 min will be conducted at 6 months, and at 12 months to explore participants' perceived responses to the intervention in the short and long term. A semistructured interview guide will be designed to address the aims of the process evaluation with probes to obtain rich description. Discussions will be digitally recorded and professionally transcribed verbatim. The interviewer will document reflective notes after each interview related to new or recurring comments, emotional responses or emerging themes. Using a directed content analysis approach ${ }^{82}$ guided by the GO-OUT conceptual framework (figure 1) and behaviour change theories underpinning the intervention, ${ }^{31}$ authors NMS, RB and JR and the qualitative research analyst will independently code 2-3 transcripts and meet to discuss and develop a coding scheme. The qualitative research analyst will then apply the coding scheme to the remaining transcripts using NVivo V.11.0 software. Authors NMS, RB and JR will review and cluster similar codes to identify emerging categories. Relationships between categories will be explored to identify themes. An audit trail will be maintained, and quotes will be used to support themes. The research team will review and confirm the themes. Triangulation will be achieved through collection and comparison of data from different sources at different sites.

\section{Patient and public involvement}

Two levels of patient/public involvement informed the design of the GO-OUT study. First, in a study of community ambulation after stroke, ${ }^{9}$ people with stroke participating in a focus group described barriers and facilitators to walking outdoors in the community and their needs and preferences for outdoor walking programmes. Findings informed the design (eg, need for increasing challenges) and location (eg, use of local parks with available benches and washrooms) of the outdoor walking programme in the GO-OUT intervention. Aspects of community ambulation that focus group participants considered as important (ie, outdoor walking, endurance, walking speed, mood and perception of health) guided formation of the research question and selection of study outcomes. Second, the GO-OUT pilot study ${ }^{31}$ involved focus groups and interviews with participants from the experimental and control groups. Participants outlined the benefits and challenges they experienced engaging with the interventions and confirmed the acceptability of the content and duration of evaluations and interventions. Findings were used to finalise the current protocol. ${ }^{31}$

We will use an infographic to share results from this study with participants. If participants are interested, they can receive reports following each evaluation that summarise the values and meaning of their scores on study measures.

\section{Sample size}

Calculations of the required sample size were conducted for our primary analysis. Based on our pilot study results, we expect a between-group difference of $25 \mathrm{~min}$ per week $(\mathrm{SD}=50)$ at 3 months, yielding an ES of 0.5. A similar ES is expected at 5.5 months assuming weather will not limit walking outdoors. Based on accelerometry/GPS data that we collected 6 months postbaseline during cooler weather conditions, a decrease in ES of $\sim 10 \%$ to 0.4 (between group difference $20 \mathrm{~min}$ per week, $\mathrm{SD}=50$ ) is expected for the $0-12$ month comparison.

A 20 min group difference exceeds $10 \%$ of the weekly physical activity recommendation and would help move seniors from a sedentary to a low active classification associated with higher HRQL. ${ }^{83}$ Thus, sample size estimation will be based on detecting the smaller ES of 0.4. In the pilot study, there was no attrition from 0 months to 6 months; however, we have allowed for a $5 \%$ attrition rate from 0 months to 6 months in the proposed study, and a $20 \%$ attrition rate from 0 months to 12 months based on rates observed in studies of group-based physical activity interventions. ${ }^{84}$ Given an ES of 0.4, type I error level $=0.05$, type II error level=0.20, equal number of participants/ group and a $20 \%$ attrition rate, a total sample size of 240 is required. We will aim to recruit 36 people/site in year 1 and 24 people/site in year 2 to provide group sizes in the outdoor walkingprogramme that will enable a 3:1 ratio of participants-to-facilitators. Randomisation will, on average, yield study groups that are similar in known and unknown confounders. Therefore, we have not adjusted for covariates in conducting our power analysis.

\section{Recruitment}

Recruitment strategies include placing paper and electronic advertisements in newspapers, senior's centres, residences and organisations, and condition-specific organisations (eg, Arthritis Society); radio announcements and interviews; and presenting to seniors groups and classes at community/fitness centres. Interested individuals are asked to contact the site study coordinator by email or phone. The study coordinator provides an overview of the study, screens eligibility criteria with the exception of postural hypotension and resting HR and completes the Get Active Questionnaire ${ }^{85-87}$ with participants to identify any contraindications to exercise. Individuals passing this initial screen are invited to an in-person evaluation at which 
eligibility screening of postural hypotension and resting HR is completed. Eligible individuals are asked to provide written informed consent (online supplementary appendix A includes a sample consent form), sign a liability waiver (at three sites) indicating they have consulted with a health professional regarding their participation in the GO-OUT intervention and that they are not aware of any concerns regarding their participation and complete the baseline evaluation. At one site, instead of signing the waiver, the completed Get Active Questionnaire is faxed to the participant's physician who is asked to contact the study team if there are concerns about the person exercising. If there is no contact from the physician, and the person is eligible, he or she is asked to provide written informed consent.

\section{Stratification and randomisation}

Participants are stratified by site because of the concern that protocol implementation may vary across sites despite standardised study materials, protocols and training. To enable spouses or two friends (called a dyad) to be assigned to the same group, participants are stratified within each site by participant type (individual vs dyad) prior to being block randomised to either the workshop plus outdoor walking group (the GO-OUT intervention) or the workshop plus reminders group. A Toronto-based administrator, not involved with recruitment or evaluations, configured REDCap, ${ }^{88}$ the online data management system, to perform stratification and blocked randomisation (randomly ordered block sizes of 2 and 4). Treatment allocation is done once individuals have provided informed consent and have completed the baseline evaluation. Site coordinators, unaware of block sizes, then register the participant in REDCap, enter data on the stratification variables and obtain the group assignment.

\section{Quantitative data analysis}

\section{Analysis plan}

We will use a mixed-effects multivariable regression model ${ }^{89}$ to test for differences between the GO-OUT intervention group and the WR group for the primary and secondary endpoints across measurement occasions. Specifically, the model will include a random intercept, and fixed effects of group, time (categorical variable) and their two-way interaction. Linear contrasts will be used to test for differences between the groups in the difference between the baseline and 3-month, baseline and 5.5-month and baseline and 12-month measurement occasions. These tests will be conducted using a likelihood ratio test, which asymptotically follows a $\chi^{2}$ distribution. When testing for a difference between groups between the baseline and 3-month occasions, we will also evaluate the effect of adjusting for each stratification variable (site and participant type) separately. These assessments will also be conducted using a likelihood ratio test. If adjustment improves the precision of estimation, then we will adjust for each variable in all models. ${ }^{90}$ We will compare baseline demographic and clinical characteristics of participants in the experimental and control groups using t-tests for continuous measures and $\chi^{2}$ tests of independence for categorical variables. Variables that differ between groups $(\alpha=0.05)$ will be entered into all multivariable linear regression models as covariates. The primary analysis will be intention-to-treat with a secondary per protocol analysis. The primary and secondary study aims and hypotheses are as follows:

\section{Primary aims}

To test the short-term and long-term effects of the GO-OUT intervention compared with the WR intervention on outdoor walking activity.

\section{Primary hypotheses}

The change in average number of minutes per week spent walking outdoors from baseline to 3 months, baseline to 5.5 months and baseline to 12 months will be significantly higher in individuals who complete the GO-OUT intervention than in those who complete the WR programme.

\section{Secondary aim 1}

To test whether initial frailty level modifies the effect of the GO-OUT intervention compared with the WR intervention on outdoor walking activity.

\section{Secondary hypothesis 1}

Individuals who are prefrail will demonstrate greater change in the average number of minutes spent walking outdoors per week from baseline to 3, 5.5 and 12 months than those who are frail or robust.

\section{Secondary aim 2}

To test the short-term and long-term effects of the GO-OUT intervention compared with the WR intervention on self-reported outdoor walking, physical activity, lifespace mobility, participation, HRQL, balance, leg strength, walking self-efficacy, walking speed, walking endurance, emotional health, HR and BP.

\section{Hypotheses for secondary aim 2}

The change in mean self-reported time per week spent walking outdoors (CHAMPS); mean time spent in moderate- to vigorous-intensity aerobic physical activity (accelerometry and CHAMPS); mean life space assessment score; mean time spent per week participating in meaningful activity (CHAMPS total score); mean PGI score; and mean score on the mini-BESTest, sit-to-stand test, ambulatory self-confidence questionnaire, $10 \mathrm{~m}$ and $6 \mathrm{~min}$ walk tests and emotional well-being scale of the RAND-36, from baseline to 3 months, baseline to 5.5 months and baseline to 12 months will be significantly higher (and mean HR and systolic and diastolic BP will be significantly lower) in individuals who complete the GO-OUT intervention than in those who complete the WR intervention.

\section{Study organisation and management}

NMS leads the central coordinating centre in Toronto and with $\mathrm{RB}$ is responsible for all aspects of trial 
implementation. CAJ, RB, NMS and NM are site investigators for the Edmonton, Winnipeg, Toronto and Montreal sites, respectively, and with a site coordinator, oversee study implementation at that site. Site personnel share non-confidential research materials using Dropbox (www.Dropbox.com). REDCap, ${ }^{88}$ a centralised data management software, is used for secure data collection and dispensation of randomised intervention assignments. Toronto-based coordinators monitor data entry in REDCap and report on data quality to sites after each evaluation. SW supervises quality and processing of the ActiGraph and GPS data at the Winnipeg site. LL and $\mathrm{JR}$ will oversee the quantitative and qualitative data analyses, respectively, in collaboration with principal investigators. Study investigators may request access to a study dataset from principal investigators NMS and RB.

\section{Ethics and dissemination}

A Data Safety Monitoring Board (DSMB) will monitor adverse events. Falling is the primary concern in this study given that people are being asked to walk outdoors. Thus, we have developed a stopping rule based on the occurrence of falls related to the GO-OUT study that result in a serious adverse event. A fall related to the GO-OUT study is defined as one that occurs during the study evaluations, ${ }^{91}$ study interventions (ie, workshop and outdoor walking group sessions), ${ }^{91}$ or while walking outdoors for exercise. A serious adverse event is one that leads to death, a life-threatening adverse event, inpatient hospitalisation or a persistent or significant disability or incapacity that lasts more than 48 hours and limits activities of daily living. ${ }^{91}$

The reported rate of injurious falls measured in community dwelling older adults in Canada of $7 \%{ }^{93}$ will be used as an estimate of the rate of falls that result in a serious adverse event. If an individual experiences multiple falls related to the GO-OUT study that result in a serious adverse event, each fall will be counted. If the rate of falls related to the GO-OUT study that result in a serious adverse event exceeds $7 \%$ of the total number of participants randomised in the study, then the DSMB will discuss whether there is sufficient evidence to stop the trial across all sites. For example, if 240 participants are randomised, and the number of falls related to the GO-OUT study that result in a serious adverse event exceeds 17 (7\% of 240), we will consider stopping the trial. This decision will be made by the principal investigators, in consultation with the DSMB and coinvestigators. The DSMB is composed of three researchers with expertise in the rehabilitation and investigation of walking in older adults and who are free of conflict of interest. The DSMB meets quarterly to review adverse events, including falls and injurious falls, to assess whether the trial should be halted.

We plan to share study findings through lay summaries, conference presentations and publications in peer-reviewed journals. If findings are positive, we will develop materials and educational initiatives in collaboration with the Canadian Society for Exercise Physiology to facilitate implementation of the GO-OUT intervention.

\section{DISCUSSION}

This multisite RCT is designed to evaluate the impact of an educational workshop and 10-week outdoor walking programme compared with the workshop and weekly reminders for 10 weeks on increasing the level of outdoor walking activity in older adults who have difficulty walking outdoors. Limited outdoor walking is a sign of frailty and can leave older adults feeling a sense of social isolation. Persistent sedentary behaviour among older adults places them at risk of further decline in mobility, which can lead to a decrease in functional independence, caregiver burden and, ultimately, loss of independent living. ${ }^{3}{ }^{4}$ Community-based exercise programmes that provide contextually relevant opportunities to practice outdoor walking have the potential to offset the negative consequences of mobility limitations and sedentary behaviour. The results of this trial will contribute to our understanding of the role of theory-based, task-oriented training programmes led by rehabilitation professionals for community-dwelling older adults.

The multisite nature of this study will enable us to test the value of the intervention in community-dwelling older adults with a variety of comorbid conditions and mobility levels. It will allow us to characterise individuals who are interested in this type of programme and increase understanding of the effect of the intervention on secondary outcomes related to physical function, cardiovascular risk factors and HRQL. The trial will enable us to examine whether baseline level of frailty modifies the effect of the intervention to understand whether the intervention may be more or less potent in people with different levels of frailty. We will be able to evaluate the influence of neighbourhood walkability on participants' engagement in outdoor walking activity.

By comparing the GO-OUT and WR interventions, the trial will increase our understanding of the relative importance of organised and scheduled walking practice compared with weekly prompting to motivate individuals to walk outdoors. The interventions will ideally be delivered in summer months. However, the qualitative component of this study will enable exploration of older adults' preferences for wintertime programmes that would help them to maintain the capacity to walk outdoors during the winter months. Finally, use of the Framework for Enhancing the Value of Research for Dissemination and Implementation ${ }^{33}$ will help us to understand the applicability of study findings to real-world settings.

\section{Author affiliations}

${ }^{1}$ Department of Physical Therapy, University of Toronto, Toronto, Ontario, Canada

${ }^{2}$ Toronto Rehabilitation Institute, University Health Network, Toronto, Ontario, Canada ${ }^{3}$ Department of Physical Therapy, University of Manitoba, Winnipeg, Manitoba,

Canada

${ }^{4}$ Department of Physical Therapy, University of Alberta, Edmonton, Alberta, Canada 
${ }^{5}$ School of Physical and Occupational Therapy, McGill University, Montreal, Quebec, Canada

${ }^{6}$ Department of Community Health Sciences, University of Manitoba, Winnipeg, Manitoba, Canada

${ }^{7}$ Department of Occupational Therapy, University of Manitoba, Winnipeg, Manitoba, Canada

${ }^{8}$ Bruyere Research Institute, Ottawa, Ontario, Canada

${ }^{9}$ Section of Geriatric Medicine, University of Manitoba, Winnipeg, Manitoba, Canada

${ }^{10}$ College of Kinesiology, University of Saskatchewan, Saskatoon, Saskatchewan, Canada

Contributors NMS co-led the conceptualisation, design and implementation of this research protocol. She was the primary author for this manuscript. RB co-led the conceptualisation, design and implementation of this research protocol. She is a contributing author for this manuscript. SCW led the development of the data collection and analysis plan for the primary outcome measure and contributed to the conceptualisation and design of this research protocol. She is a contributing author for this manuscript. CAJ contributed to the design and implementation of this research protocol. She is a contributing author for this manuscript. NEM contributed to the conceptualisation, design and implementation of this research protocol. She is a contributing author for this manuscript. LML led the development of the statistical analysis plan and contributed to the design of this research protocol. She is a contributing author for this manuscript. JR led the development of the qualitative data analysis plan and contributed to the design of this research protocol. She is a contributing author for this manuscript. TG contributed to the design of this research protocol. She is a contributing author for this manuscript. $\mathrm{Cvl}$ led the development of the falls risk screening plan and contributed to the design of this research protocol. She is a contributing author for this manuscript. PDC represents the study sponsor, the Canadian Society for Exercise Physiology ( www.csep.ca). He contributed to the design of this research protocol and led development of the dissemination plan. He is a contributing author for this manuscript.

Funding This work is supported by the Canadian Institutes of Health Research, grant number 376439. NMS is supported by a Heart and Stroke Foundation Mid-Career Investigator Award.

Competing interests None declared.

Patient consent for publication Not required.

Ethics approval Research ethics boards at the University of Toronto, University of Manitoba, University of Alberta and McGill University have approved the study protocol.

Provenance and peer review Not commissioned; peer reviewed for ethical and funding approval prior to submission.

Open access This is an open access article distributed in accordance with the Creative Commons Attribution Non Commercial (CC BY-NC 4.0) license, which permits others to distribute, remix, adapt, build upon this work non-commercially, and license their derivative works on different terms, provided the original work is properly cited, appropriate credit is given, any changes made indicated, and the use is non-commercial. See: http://creativecommons.org/licenses/by-nc/4.0/.

\section{REFERENCES}

1. Statistics Canada. Canadian Community Health Survey: Walking Data. 2009.

2. Rantakokko M, Mänty M, Iwarsson S, et al. Fear of moving outdoors and development of outdoor walking difficulty in older people. J Am Geriatr Soc 2009;57:634-40.

3. Fujita K, Fujiwara Y, Chaves PH, et al. Frequency of going outdoors as a good predictors for incident disability of physical function as well as disability recovery in community-dwelling older adults in rural Japan. J Epidemiol 2006;16:261-70.

4. Sirois MJ, Émond M, Ouellet MC, et al. Cumulative incidence of functional decline after minor injuries in previously independent older Canadian individuals in the emergency department. J Am Geriatr Soc 2013;61:1661-8.

5. Gardener EA, Huppert FA, Guralnik JM, et al. Middle-aged and mobility-limited: prevalence of disability and symptom attributions in a national survey. J Gen Intern Med 2006;21:1091-6.

6. King AC, Sallis JF, Frank LD, et al. Aging in neighborhoods differing in walkability and income: associations with physical activity and obesity in older adults. Soc Sci Med 2011;73:1525-33.
7. Sessford JD, Jung M, Brawley LR, et al. Do older adults' beliefs about their community mobility predict walking performance? J Aging Phys Act 2015;23:272-8.

8. Shumway-Cook A, Patla AE, Stewart A, et al. Environmental demands associated with community mobility in older adults with and without mobility disabilities. Phys Ther 2002;82:670-81.

9. Barclay R, Ripat J, Mayo N. Factors describing community ambulation after stroke: a mixed-methods study. Clin Rehabil 2015;29:509-21.

10. Patla AE, Shumway-Cook A. Dimensions of mobility: defining the complexity and difficulty associated with community mobility. $J$ Aging Phys Act 1999;7:7-19.

11. Ripat JD, Redmond JD, Grabowecky BR. The winter walkability project: occupational therapists' role in promoting citizen engagement. Can J Occup Ther 2010;77:7-14.

12. Kassavou A, Turner A, French DP. Do interventions to promote walking in groups increase physical activity? A meta-analysis. Int $J$ Behav Nutr Phys Act 2013;10:18.

13. Kerr J, Rosenberg DE, Nathan A, et al. Applying the ecological model of behavior change to a physical activity trial in retirement communities: description of the study protocol. Contemp Clin Trials 2012;33:1180-8.

14. Whitt-Glover MC, Goldmon MV, Karanja N, et al. Learning and Developing Individual Exercise Skills (L.A.D.I.E.S.) for a better life: a physical activity intervention for black women. Contemp Clin Trials 2012;33:1159-71.

15. Leung W, Ashton T, Kolt GS, et al. Cost-effectiveness of pedometerbased versus time-based Green Prescriptions: the Healthy Steps Study. Aust J Prim Health 2012;18:204-11.

16. Foreman R, van Uffelen JG, Brown WJ. Twelve month impact of the Just Walk It program on physical activity levels. Health Promot $J$ Austr 2012;23:101-7.

17. Pereira MA, Kriska $A M$, Day $R D$, et al. A randomized walking trial in postmenopausal women: effects on physical activity and health 10 years later. Arch Intern Med 1998;158:1695-701.

18. Brosseau L, Wells GA, Kenny GP, et al. The implementation of a community-based aerobic walking program for mild to moderate knee osteoarthritis (OA): a knowlege translation (KT) randomized controlled trial $(\mathrm{RCT})$ : part I: the uptake of the Ottawa panel clinical practice guidelines (CPGs). BMC Public Health 2012;12:871.

19. Bruno M, Cummins S, Gaudiano L, et al. Effectiveness of two arthritis foundation programs: walk with ease, and you can break the pain cycle. Clin Interv Aging 2006;1:295-306.

20. Barclay R, Stevenson T, Poluha W, et al. Interventions for improving community ambulation in individuals with stroke (review). Cochrane Libr 2015.

21. Shumway-Cook A, Woollacott MH. Motor control: translating research into clinical practice. Philadelphia: Wilkins, 2012.

22. VanSwearingen JM, Perera S, Brach JS, et al. Impact of exercise to improve gait efficiency on activity and participation in older adults with mobility limitations: a randomized controlled trial. Phys Ther 2011;91:1740-51

23. Figueiredo S, Finch L, Mai J, et al. Nordic walking for geriatric rehabilitation: a randomized pilot trial. Disabil Rehabil 2013;35:968-75.

24. Parkatti T, Perttunen J, Wacker P. Improvements in functional capacity from Nordic walking: a randomized-controlled trial among elderly people. J Aging Phys Act 2012;20:93-105.

25. Tschentscher M, Niederseer D, Niebauer J. Health benefits of Nordic walking: a systematic review. Am J Prev Med 2013;44:76-84.

26. Duvall J, De Young R. Some strategies for sustaining a walking routine: insights from experienced walkers. J Phys Act Health 2013;10:10-18.

27. Kassavou A, French DP, Chamberlain K. How do environmental factors influence walking in groups? A walk-along study. J Health Psychol 2015;20:1328-39.

28. Isaacs AJ, Critchley JA, Tai SS, et al. Exercise Evaluation Randomised Trial (EXERT): a randomised trial comparing GP referral for leisure centre-based exercise, community-based walking and advice only. Health Technol Assess 2007;11:1-165.

29. Ridgers ND, Fairclough S. Assessing free-living physical activity using accelerometry: Practical issues for researchers and practitioners. Eur J Sport Sci 2011;11:205-13.

30. Kerr J, Marshall S, Godbole S, et al. The relationship between outdoor activity and health in older adults using GPS. Int J Environ Res Public Health 2012:9:4615-25.

31. Barclay R, Webber S, Ripat J, et al. Safety and feasibility of an interactive workshop and facilitated outdoor walking group compared to a workshop alone in increasing outdoor walking 
activity among older adults: a pilot randomized controlled trial. Pilot Feasibility Stud 2018;4:4

32. Statistics Canada. Census Profile, 2016 Census. 2016. https:// www12.statcan.gc.ca/census-recensement/2016/dp-pd/prof/index. $\mathrm{cfm}$ ? Lang=E

33. Neta G, Glasgow RE, Carpenter CR, et al. A framework for enhancing the value of research for dissemination and implementation. Am J Public Health 2015;105:49-57.

34. Moher D, Hopewell S, Schulz KF, et al. CONSORT 2010 explanation and elaboration: updated guidelines for reporting parallel group randomised trials. BMJ 2010;340:c869.

35. The SPIRIT Group. The SPIRIT Statement. 2018 http://www.spiritstatement.org/spirit-statement/ (Accessed 24 December 2018).

36. Roccaforte $\mathrm{WH}$, Burke WJ, Bayer BL, et al. Validation of a telephone version of the mini-mental state examination. J Am Geriatr Soc 1992;40:697-702.

37. Panel on Prevention of Falls in Older Persons, American Geriatrics Society and British Geriatrics Society. Summary of the Updated American Geriatrics Society/British Geriatrics Society clinical practice guideline for prevention of falls in older persons. J Am Geriatr Soc 2011;59:148-57.

38. Labreche T, Nandakumar K, Althomali M, et al. Development and validation of Visual Impairment as a Risk for Falls Questionnaire. Age Ageing 2018;47:444-50.

39. Canadian Society for Exercise Physiology. Canadian physical activity guidelines for older adults $2018 \mathrm{https} / / / c s e p g u i d e l i n e s . c a /$ adults-65/ (Accessed 15 December 2018).

40. Bandura A. Health promotion by social cognitive means. Health Educ Behav 2004;31:143-64.

41. Thompson Coon J, Boddy K, Stein K, et al. Does participating in physical activity in outdoor natural environments have a greater effect on physical and mental wellbeing than physical activity indoors? A systematic review. Environ Sci Technol 2011:45:1761-72

42. Aadland E, Ylvisåker E. Reliability of objectively measured sedentary time and physical activity in adults. PLoS One 2015;10:e0133296.

43. Wetten AA, Batterham M, Tan SY, et al. Relative validity of 3 accelerometer models for estimating energy expenditure during light activity. J Phys Act Health 2014;11:638-47.

44. Rabinovich RA, Louvaris Z, Raste Y, et al. PROactive Consortium. Validity of physical activity monitors during daily life in patients with COPD. Eur Respir J 2013;42:1205-15.

45. Qstarz International. GPS Travel Recorder. 2013 http://www.qstarz. com/Products/GPS\%20Products/BT-Q1000XT-F.htm (Accessed 24 December 2018).

46. Troiano RP, Berrigan D, Dodd KW, et al. Physical activity in the United States measured by accelerometer. Med Sci Sports Exerc 2008;40:181-8.

47. Matthew CE. Calibration of accelerometer output for adults. Med Sci Sports Exerc 2005;37(11 Suppl):S512-22.

48. Tudor-Locke C, Camhi SM, Leonardi C, et al. Patterns of adult stepping cadence in the 2005-2006 NHANES. Prev Med 2011:53:178-81.

49. Holland AE, Spruit MA, Troosters T, et al. An official European Respiratory Society/American Thoracic Society technical standard: field walking tests in chronic respiratory disease. Eur Respir J 2014:44:1428-46.

50. American College of Sports Medicine. ACSM's Resources for the personal trainer. 3rd ed. Philadelphia, PA: Lippincott Williams \& Wilkins, 2010.

51. Steffen TM, Hacker TA, Mollinger L. Age- and gender-related test performance in community-dwelling elderly people: Six-Minute Walk Test, Berg Balance Scale, Timed Up \& Go Test, and gait speeds. Phys Ther 2002;82:128-37.

52. Ng SS, Hui-Chan CW. The timed up \& go test: its reliability and association with lower-limb impairments and locomotor capacities in people with chronic stroke. Arch Phys Med Rehabil 2005;86:1641-7.

53. Horak FB, Wrisley DM, Frank J. The balance evaluation systems test (BESTest) to differentiate balance deficits. Phys Ther 2009;89.

54. King L, Horak F. On the mini-BESTest: scoring and the reporting of total scores. Phys Ther 2013;93:571-5.

55. Godi M, Franchignoni F, Caligari M, et al. Comparison of reliability, validity, and responsiveness of the mini-BESTest and Berg Balance Scale in patients with balance disorders. Phys Ther 2013:93:158-67.

56. Rikli RE, Jones CJ. Development and validation of a functional fitness test for community-residing older adults. J Aging Phys Act 1999;7:129-61.
57. Macfarlane DJ, Chou KL, Cheng YH, et al. Validity and normative data for thirty-second chair stand test in elderly communitydwelling Hong Kong Chinese. Am J Hum Biol 2006;18:418-21.

58. Asano M, Miller WC, Eng JJ. Development and psychometric properties of the ambulatory self-confidence questionnaire. Gerontology 2007;53:373-81.

59. Perera S, Mody SH, Woodman RC, et al. Meaningful change and responsiveness in common physical performance measures in older adults. J Am Geriatr Soc 2006;54:743-9.

60. Peters DM, Fritz SL, Krotish DE. Assessing the reliability and validity of a shorter walk test compared with the 10-Meter Walk Test for measurements of gait speed in healthy, older adults. J Geriatr Phys Ther 2013;36:24-30.

61. Kim HJ, Park I, Lee HJ, et al. The reliability and validity of gait speed with different walking pace and distances against general health, physical function, and chronic disease in aged adults. $J$ Exerc Nutrition Biochem 2016;20:46-50.

62. Flansbjer UB, Holmbäck AM, Downham D, et al. Reliability of gait performance tests in men and women with hemiparesis after stroke. $J$ Rehabil Med 2005;37:75-82.

63. Baker PS, Bodner EV, Allman RM. Measuring life-space mobility in community-dwelling older adults. J Am Geriatr Soc 2003:51:1610-4.

64. Peel C, Sawyer Baker P, Roth DL, et al. Assessing mobility in older adults: the UAB Study of Aging Life-Space Assessment. Phys Ther 2005;85:1008-119.

65. Giles K, Marshall AL. Repeatability and accuracy of CHAMPS as a measure of physical activity in a community sample of older Australian adults. J Phys Act Health 2009;6:221-9.

66. Hays RD, Morales LS. The RAND-36 measure of health-related quality of life. Ann Med 2001;33:350-7.

67. Ruta DA, Garratt AM, Leng M, et al. A new approach to the measurement of quality of life. The Patient-Generated Index. Med Care 1994;32:1109-26.

68. Martin F, Camfield L, Rodham K, et al. Twelve years' experience with the Patient Generated Index (PGI) of quality of life: a graded structured review. Qual Life Res 2007;16:705-15.

69. Fried LP, Tangen CM, Walston J, et al. Cardiovascular Health Study Collaborative Research Group. Frailty in older adults: evidence for a phenotype. J Gerontol A Biol Sci Med Sci 2001;56:M146-M157.

70. Saelens BE, Sallis JF, Black JB, et al. Neighborhood-based differences in physical activity: an environment scale evaluation. Am J Public Health 2003;93:1552-8.

71. Cerin E, Saelens BE, Sallis JF, et al. Neighborhood Environment Walkability Scale: validity and development of a short form. Med Sci Sports Exerc 2006;38:1682-91.

72. Cerin E, Conway TL, Cain KL, et al. Sharing good NEWS across the world: developing comparable scores across 12 countries for the Neighborhood Environment Walkability Scale (NEWS). BMC Public Health 2013;13:309.

73. Charlson ME, Pompei P, Ales KL, et al. A new method of classifying prognostic comorbidity in longitudinal studies: development and validation. J Chronic Dis 1987;40:373-83.

74. Quan H, Li B, Couris CM, et al. Updating and validating the Charlson comorbidity index and score for risk adjustment in hospital discharge abstracts using data from 6 countries. Am J Epidemiol 2011;173:676-82.

75. Roffman CE, Buchanan J, Allison GT. Charlson comorbidities index J Physiother 2016;62:171.

76. Lamb SE, Jorstad-Stein EC, Hauer K, et al. Development of a common outcome data set for fall injury prevention trials: the Prevention of Falls Network Europe consensus. J Am Geriatr Soc 2005;53:1618-22.

77. Muir SW, Berg K, Chesworth B, et al. Balance impairment as a risk factor for falls in community-dwelling older adults who are high functioning: a prospective study. Phys Ther 2010;90:338-47.

78. Ferm L, Rasmussen CDN, Jørgensen MB. Operationalizing a mode to quantify implementation of a multi-component intervention in a stepped-wedge trial. Implement Sci 2018;13:26.

79. Craig P, Dieppe P, Macintyre S, et al. Medical Research Council Guidance. Developing and evaluating complex interventions: the new Medical Research Council guidance. BMJ 2008;337(a165 5):a1655.

80. Lewin S, Glenton C, Oxman AD. Use of qualitative methods alongside randomised controlled trials of complex healthcare interventions: methodological study. BMJ 2009;339:b3496.

81. Bradshaw C, Atkinson S, Doody O. Employing a Qualitative Description Approach in Health Care Research. Glob Qual Nurs Res 2017;4:233339361774228.

82. Hsieh HF, Shannon SE. Three approaches to qualitative content analysis. Qual Health Res 2005;15:1277-88. 
83. Dondzila CJ, Gennuso KP, Swartz AM, et al. Dose-response walking activity and physical function in older adults. J Aging Phys Act 2015;23:194-9.

84. Yeom HA, Keller C, Fleury J. Interventions for promoting mobility in community-dwelling older adults. J Am Acad Nurse Pract 2009;21:95-100.

85. Canadian Society for Exercise Physiology. Get active questionnaire 2017 http://csep.ca/en/publications/get-active-questionnaire.

86. Petrella AFM, Gill DP, Petrella RJ. Evaluation of the Get Active Questionnaire in community-dwelling older adults. Appl Physiol Nutr Metab 2018;43:587-94.

87. Stathokostas L, Petrella AFM, Blunt W, et al. Perceptions of exercise screening among older adults. Appl Physiol Nutr Metab 2018;43:580-6.

88. Harris PA, Taylor R, Thielke R, et al. Research electronic data capture (REDCap)--a metadata-driven methodology and workflow process for providing translational research informatics support. $J$ Biomed Inform 2009;42:377-81.

89. Diez-Roux AV. Multilevel analysis in public health research. Annu Rev Public Health 2000;21:171-92.

90. Piantadosi S. Clinical trials: a methodological perspective. John Wilely \& Sons 2005

91. Duncan PW, Sullivan KJ, Behrman AL, et al. LEAPS Investigative Team. Protocol for the Locomotor Experience Applied Poststroke (LEAPS) trial: a randomized controlled trial. BMC Neurol 2007;7:39.

92. Health Canada. Guidance document: good clinical practice: integrated addendum to E6(R1) ICH topic E6(R2). 2017 https:// www.canada.ca/en/health-canada/services/drugs-healthproducts/drug-products/applications-submissions/guidancedocuments/efficacy/guidance-document-good-clinical-practiceintegrated-addendum-e6-r1-topic-e6-r2.html (Accessed December 3, 2018).

93. Handrigan GA, Maltais N, Gagné M, et al. Sex-specific association between obesity and self-reported falls and injuries among community-dwelling Canadians aged 65 years and older. Osteoporos Int 2017;28:483-94.
94. Logan PA, Gladman JR, Avery A, et al. Randomised controlled trial of an occupational therapy intervention to increase outdoor mobility after stroke. BMJ 2004;329:1372 https://doi.org/.

95. Mayo NE, Anderson S, Barclay R, et al. Getting on with the rest of your life following stroke: a randomized trial of a complex intervention aimed at enhancing life participation post stroke. Clin Rehabil 2015;29:1198-211.

96. Ajzen I. The theory of planned behavior. Organ Behav Hum Decis Process 1991;50:179-211.

97. Talbot LA, Gaines JM, Huynh TN, et al. A home-based pedometerdriven walking program to increase physical activity in older adults with osteoarthritis of the knee: a preliminary study. J Am Geriatr Soc 2003;51:387-92.

98. Tudor-Locke C, Craig CL, Aoyagi Y, et al. How many steps/day are enough? For older adults and special populations. Int J Behav Nutr Phys Act 2011;8:80.

99. Marshall SJ, Levy SS, Tudor-Locke CE, et al. Translating physical activity recommendations into a pedometer-based step goal: 3000 steps in 30 minutes. Am J Prev Med 2009;36:410-5.

100. Helfand AE. Basic considerations for geriatric footwear. Clin Podiatr Med Surg 2003;20:593-605.

101. Arbour KP, Ginis KAM. Helping middle-aged women translate physical activity intentions into action: combining the theory of planned behavior and implementation intentions. J Appl Biobehav Res 2004;9:172-87.

102. Borg GA. Psychophysical bases of perceived exertion. Med Sci Sports Exerc 1982;14:377???381-81.

103. MacKay-Lyons M, Thornton M. e-Aerobics. 2018 https://www. strokengine.ca/en/e-learning/ (Accessed March 31 2018).

104. Winnipeg Regional Health Authority. Staying on your feet: taking steps to prevent falls. 2015 www.preventfalls.ca (Accessed December 24 2018).

105. Nelson ME, Rejeski WJ, Blair SN, et al. American College of Sports MedicineAmerican Heart Association. Physical activity and public health in older adults: recommendation from the American College of Sports Medicine and the American Heart Association. Circulation 2007;116:1094. 